\title{
Erratum for: miR-26a-5p protects against myocardial ischemia/reperfusion injury by regulating the PTEN/ PI3K/AKT signaling pathway
}

\author{
Xiaowei Xing (i) ${ }^{1}$, Shuang Guo ${ }^{2}{ }^{2}$, Guanghao Zhang (ii) ${ }^{1}$, Yusheng Liu (ii) ${ }^{1}$, Shaojie Bi (i) ${ }^{1}$, \\ Xin Wang (ii) ${ }^{1 *}$, and Qinghua Lu(i) ${ }^{1 *}$ \\ ${ }^{1}$ Department of Cardiology, The Second Hospital of Shandong University, Jinan, Shandong, China \\ ${ }^{2}$ Department of Gastroenterology, The Second Hospital of Shandong University, Jinan, Shandong, China
}

Erratum for: Braz J Med Biol Res | doi: http://dx.doi.org/10.1590/1414-431X20199106

The authors notified the Editors of the Brazilian Journal of Medical and Biological Research that the internal controls of Figure $4 \mathrm{E}, 4 \mathrm{~F}$ and $4 \mathrm{G}$ were incorrectly sent for publication. Thus, the corresponding semi-quantitative analyses of protein blots had to be recalculated and the histograms must be replaced. There were also errors in the text.

\section{Text corrections and revised Figure 1 and Figure 4.}

Page 3, left column, last line: The internal control was $\beta$-actin.

Page 4, Figure $1 \mathrm{C}$ and 1D. The internal control (Actin) has been changed to $\beta$-actin.

Page 5, right column, line 8 from bottom: Figure $4 E, P=0.0001$ )

Page 7, last line of Figure 4 legend: ${ }^{\# \#} \mathrm{P}<0.001$ should be deleted (as shown in the corrected legend and Figure 4 on page 3 of this erratum). 
A

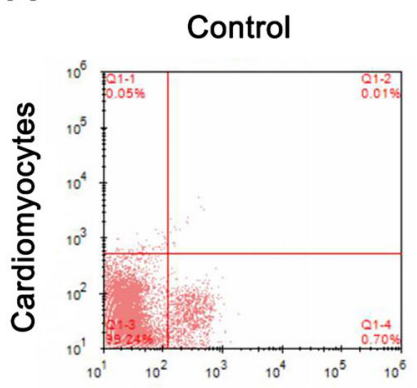

B

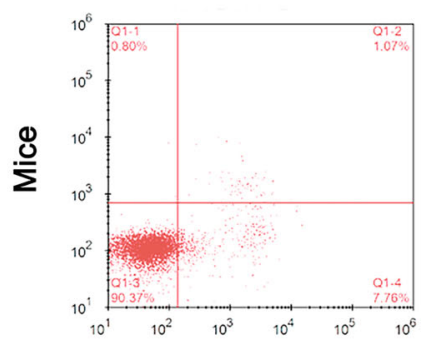

H/R treatment

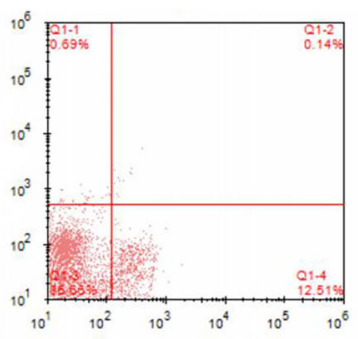

I/R treatment

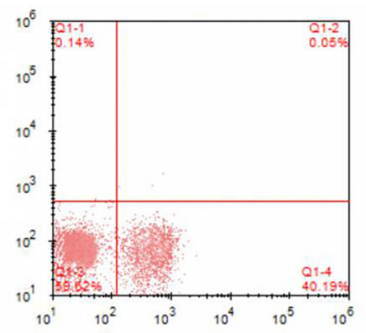

E

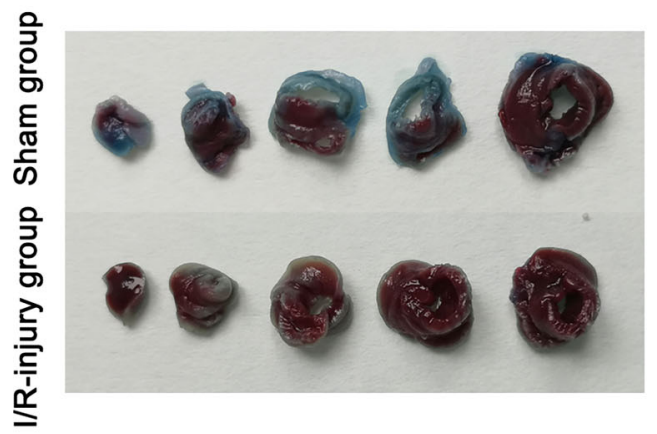

C

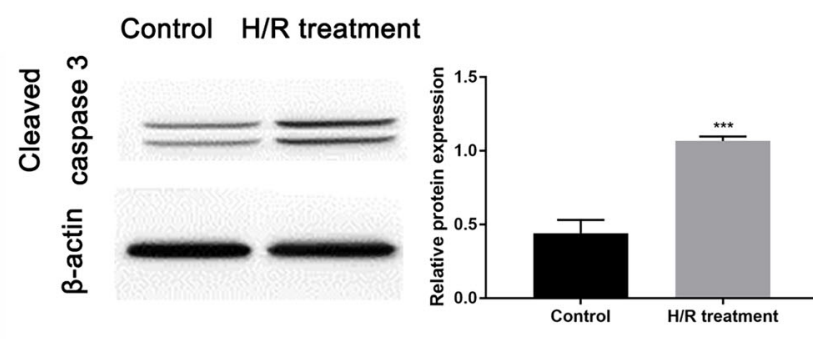

D

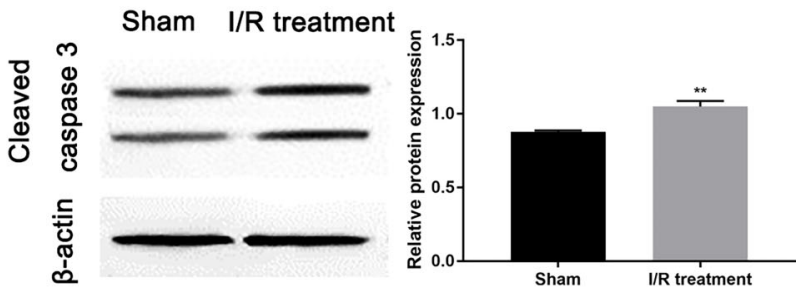

F

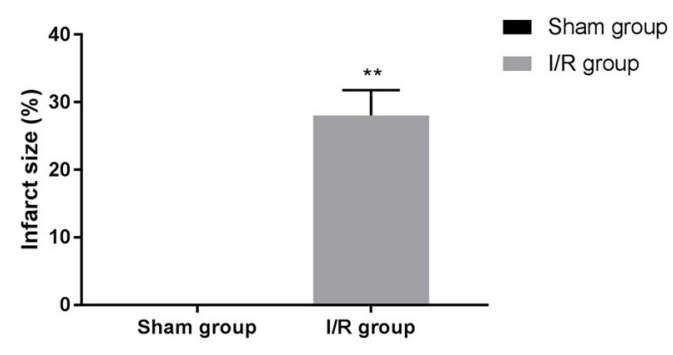

Figure 1. Establishment of ischemia/reperfusion (I/R) injury model. The images of flow cytometry show apoptosis in (A) cardiomyocytes and (B) myocardium of mice upon I/R injury. Western blot examined the expression of cleaved caspase 3 in (C) cardiomyocytes submitted to hypoxia/reoxygenation (H/R) treatment and (D) myocardial tissue upon l/R treatment. E, Representative images of Evans blue/TTC staining in five continuous slices of left ventricle from mice hearts treated with or without I/R treatment. F, The infarct size was quantified by Image-Pro Plus software. Data are reported as means $\pm \mathrm{SD}$. ${ }^{* *} \mathrm{P}<0.01,{ }^{* * *} \mathrm{P}<0.001$ vs control groups (t-test). 
A

PTEN (41-47) 5'...ACACCAUGAAAAUAAACUUGAAU...3' miR-26a-5p 3' UCGgAUAGGACCUAAUGAACUU 5'

PTEN (1261-1268) 5'...ACUGUUAGGGAAUUUUACUUGAA....3' miR-26a-5p 3' UCGgAUAGGACCUAAUGAACUU 5'

PTEN (2619-2626) 5'...UUACAUGUCUGAAGUUACUUGAA...3' miR-26a-5p 3' UGGGAUAGGACCUAAUGAACUU 5'

PTEN (3800-3807) 5'...CUAAAGGACUUUUUGUACUUGAA....3' miR-26a-5p 3' UCGGAUAGGACCUAAUGAACUU 5'

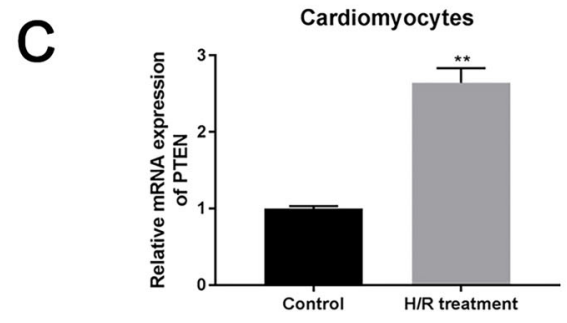

Myocardial tissue

D

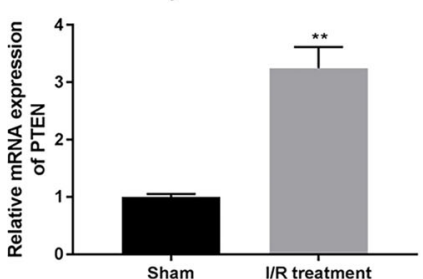

G

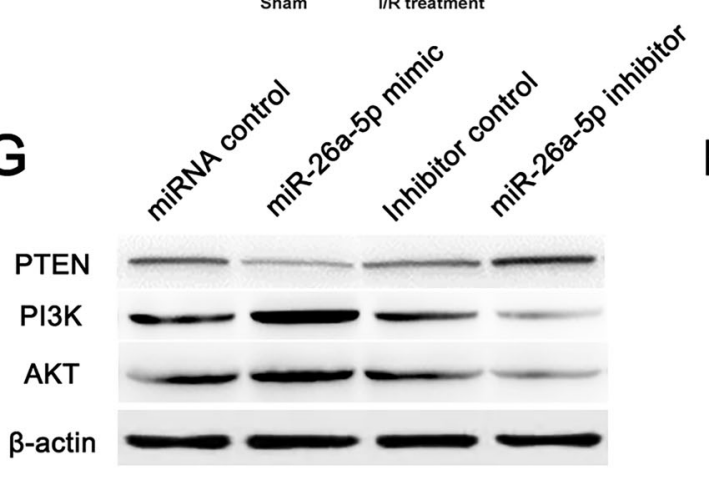

B

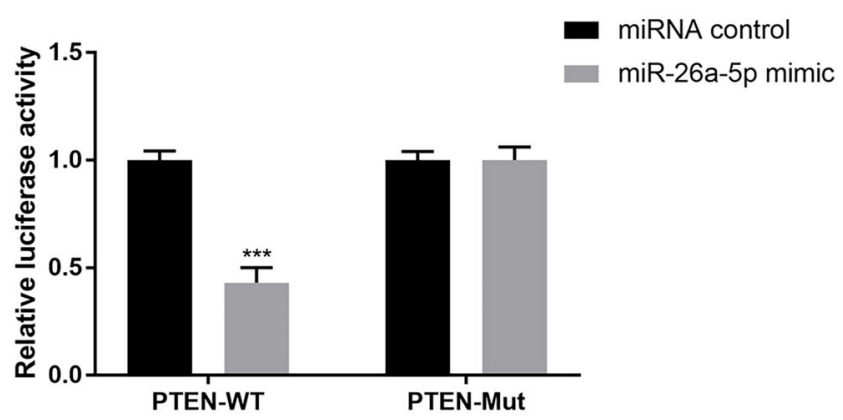

E

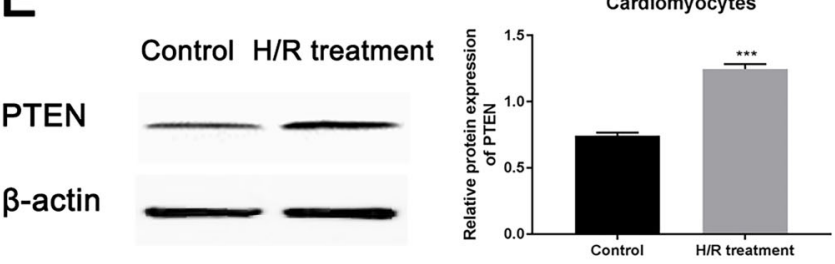

$\mathbf{F}$

Sham I/R treatment

PTEN

B-actin
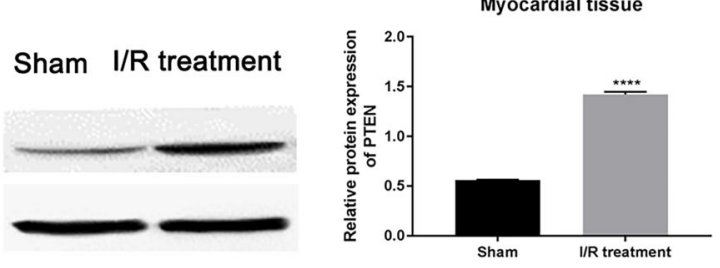

$\mathrm{H}$

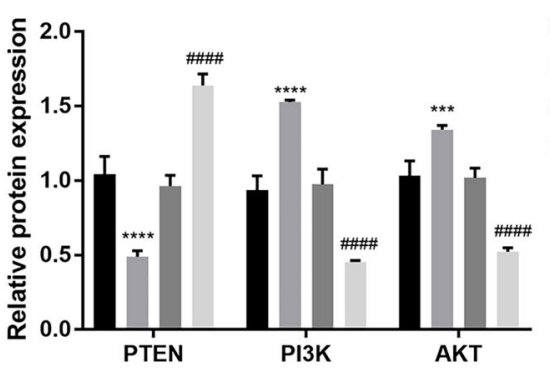

Figure 4. Interaction between miR-26a-5p and PTEN. A, Binding sites between miR-26a-5p and PTEN. B, Luciferase reporter assay measured the luciferase activity of PTEN-WT (wild type) or PTEN-Mut (mutant) vector. The mRNA and protein expression of PTEN in (C and $\mathbf{E}$ ) cardiomyocytes after hypoxia/reoxygenation $(H / R)$ and (D and $\mathbf{F})$ myocardial tissue upon ischemia/reperfusion (I/R) injury was measured by qRT-PCR and western blot, respectively. After transfection of four different miR-26a-5p vectors, the expression of PTEN, $\mathrm{PI} 3 \mathrm{~K}$, and AKT was evaluated by $(\mathbf{G})$ western blot and quantified by $(\mathbf{H})$ ImageJ software. Data are reported as means $\pm \mathrm{SD}$. ${ }^{* *} \mathrm{P}<0.01$,

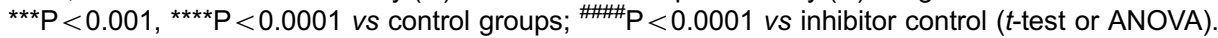




\title{
miR-26a-5p protects against myocardial ischemia/ reperfusion injury by regulating the PTEN/PI3K/AKT signaling pathway
}

\author{
Xiaowei Xing (iD ${ }^{1}$, Shuang Guo (i) $^{2}$, Guanghao Zhang (ii) ${ }^{1}$, Yusheng Liu (iD ${ }^{1}$, Shaojie Bi ${ }^{1}{ }^{1}$, \\ Xin Wang (i) ${ }^{1 *}$, and Qinghua Lu (i) ${ }^{1 *}$ \\ ${ }^{1}$ Department of Cardiology, The Second Hospital of Shandong University, Jinan, Shandong, China \\ ${ }^{2}$ Department of Gastroenterology, The Second Hospital of Shandong University, Jinan, Shandong, China
}

\begin{abstract}
Reperfusion strategies in acute myocardial infarction (AMI) can cause a series of additional clinical damage, defined as myocardial ischemia/reperfusion (I/R) injury, and thus there is a need for effective therapeutic methods to attenuate I/R injury. miR-26a-5p has been proven to be an essential regulator for biological processes in different cell types. Nevertheless, the role of miR-26a-5p in myocardial I/R injury has not yet been reported. We established an I/R injury model in vitro and in vivo. In vitro, we used cardiomyocytes to simulate $\mathrm{l} / \mathrm{R}$ injury using hypoxia/reoxygenation $(\mathrm{H} / \mathrm{R})$ assay. In vivo, we used C57BL/6 mice to construct I/R injury model. The infarct area was examined by TTC staining. The level of miR-26a-5p and PTEN was determined by bioinformatics methods, qRT-PCR, and western blot. In addition, the viability and apoptosis of cardiomyocytes were separately detected by MTT and flow cytometry. The targeting relationship between miR-26a-5p and PTEN was analyzed by the TargetScan website and luciferase reporter assay. I/R and $H / R$ treatment induced myocardial tissue injury and cardiomyocyte apoptosis, respectively. The results showed that miR-26a-5p was down-regulated in myocardial I/R injury. PTEN was found to be a direct target of miR-26a-5p. Furthermore, miR-26a-5p effectively improved viability and inhibited apoptosis in cardiomyocytes upon I/R injury by inhibiting PTEN expression to activate the PI3K/AKT signaling pathway. miR-26a-5p could protect cardiomyocytes against I/R injury by regulating the PTEN/PI3K/AKT pathway, which offers a potential approach for myocardial $\mathrm{I} / \mathrm{R}$ injury treatment.
\end{abstract}

Key words: Acute myocardial infarction; miR-26a-5p; PTEN; Apoptosis; PI3K/AKT pathway; Myocardial ischemia/reperfusion injury

\section{Introduction}

Acute myocardial infarction (AMI) is a common type of ischemic heart disease, with serious consequences in morbidity and mortality (1). Reperfusion therapy that promotes the rapid return of blood flow to the ischemic zone in the myocardium is the major procedure for the treatment of AMI (2). Although reperfusion can ameliorate myocardial function, limit infarct size, and timely and effectively reduce mortality (3), it causes extra damage to the myocardium, a phenomenon called myocardial ischemia/reperfusion (I/R) injury (4). The features of myocardial $\mathrm{I} / \mathrm{R}$ injury mainly include cardiomyocyte death, microvascular destruction, and inflammation $(3,5)$, which may induce contractile dysfunction, cardiac arrhythmias, and myocardial infarction (6). At present, there is no effective therapeutic method for myocardial I/R injury (7). Hence, it is of great clinical significance to explore the molecular mechanism of the pathological processes of myocardial I/R injury.

MicroRNAs (miRNAs), as small noncoding RNA molecules, mediate gene expression by being complementary to a site in the $3^{\prime}$ UTRs (untranslated regions) of target mRNAs, thereby resulting in mRNA degradation or translational repression (8). miRNAs are linked to many developmental and physiologic processes, mainly including cell metastasis, proliferation, apoptosis and hematopoiesis $(9,10)$. Literature suggests that the abnormal expression level of miRNAs is relevant to the pathogenesis of various diseases $(11,12)$. For instance, miR-29 is critical for regulating tissue fibrosis, which can be regarded as an

Correspondence: Qinghua Lu: <qinghualu@yeah.net>

${ }^{*}$ These authors contributed equally to this work.

Received July 26, 2019 | Accepted October 10, 2019 
intriguing therapeutic target for fibrosis diseases (13). Down-regulation of miR-146a has been reported to protect against cardiac hypertrophy and dysfunction induced by high pressure (14). miR-26a-5p acts as either a tumor promoter or suppressor in the development and progression of diverse cancers $(15,16)$. Furthermore, miR-26a-5p has been found to be abnormally expressed in several cardiovascular diseases such as AMI $(17,18)$. However, the function of miR-26a-5p in myocardial I/R injury has not been reported.

Therefore, we established an I/R injury model in vitro and in vivo. Then, we determined the expression and role of miR-26a-5p in myocardial l/R injury using bioinformatics methods and biological experiments.

\section{Material and Methods}

\section{Database and bioinformatics analysis}

Gene Expression Omnibus (GEO, https://www.ncbi. nlm.nih.gov/geo/) was searched for "heart ischemia reperfusion" and "miRNA" to identify the dataset of myocardial I/R injury-related miRNAs. Datasets with a sample size of less than 10 were excluded. Furthermore, the expression profiles of miRNAs were determined only in samples that suffered myocardial I/R injury, which was used as a key condition for selecting the dataset. Therefore, the dataset of GSE74951 deposited by Feng et al. (19) was chosen, and this dataset based on the platform of the GPL21136 includes nine myocardial $\mathrm{I} / \mathrm{R}$ injury mice and six sham mice. The data collection processes were in accordance with all regulations. The raw data of miR-26a-5p were analyzed and normalized by the Limma package in the $R$ language (version 3.4.3). The expression difference of miR-26a-5p between myocardial I/R injury mice and sham mice was calculated by Student's t-test.

In addition, the potential target of miR-26a-5p was predicted using the TargetScan website (http://www. targetscan.org/vert_72/), and PTEN, a tumor suppressor, was selected as a candidate.

\section{Cell isolation and culture}

The present work was approved by the Ethics Committee of the Second Hospital of Shandong University. All procedures were guided by the Care and Use of Laboratory Animals guidelines from the National Institutes of Health. We purchased C57BL/6 mice from Shanghai Experimental Animal Center of Chinese Academy of Sciences (China). Primary cardiomyocytes were isolated from C57BL/6 mice as previously reported (20). Briefly, ventricles were isolated from 2-day-old mice embryonic hearts, minced, and digested with $0.25 \%$ trypsin (Gibco, USA) and $0.2 \%$ collagenase type II (Sigma, USA) for two cycles. The extract was centrifuged at $45 \mathrm{~g}$ at room temperature for $5 \mathrm{~min}$, and the supernatant was removed, followed by the addition of D-Hanks solution (Sigma). The sample was centrifuged at $100 \mathrm{~g}$ at room temperature for
$5 \mathrm{~min}$, the supernatant was removed, followed by adding the culture medium to obtain cell suspension. The obtained cells were seeded and incubated in DMEM with $1 \%$ penicillin/ streptomycin, $10 \%$ fetal bovine serum (FBS) and $4 \mathrm{mM}$ L-glutamine in an incubator with $5 \% \mathrm{CO}_{2}$ at $37^{\circ} \mathrm{C}$.

\section{Cell transfection}

miR-26a-5p inhibitor, miR-26a-5p mimic, and controls (inhibitor control, mimic control) were synthesized from Thermo Fisher Scientific (USA) and Shanghai GenePharma Co., Ltd. (China). To knock down or overexpress miR-26a-5p, primary cardiomyocytes were transfected with miR-26a-5p inhibitor or mimic by Lipofectamine 3000 (Invitrogen, USA).

\section{Establishment of cardiomyocyte hypoxia/ reoxygenation model}

To simulate I/R injury in vitro, the transfected cardiomyocytes were submitted to hypoxia/reoxygenation (H/R) treatment. The transfected cardiomyocytes were incubated to approximately $80 \%$ confluence. Then, DMEM medium was removed and changed with sugar-free and serum-free medium. Next, the cells were cultured in a hypoxic incubator with the atmosphere of $5 \% \mathrm{CO}_{2}$ and $95 \% \mathrm{~N}_{2}$ for $4 \mathrm{~h}$ to simulate ischemia. Subsequently, the cells were transferred to normal DMEM containing $1 \%$ penicillin/streptomycin, 10\% FBS, and $4 \mathrm{mM} \mathrm{L-glutamine}$ in an atmosphere of $95 \%$ air and $5 \% \mathrm{CO}_{2}$ for $2 \mathrm{~h}$ to simulate reperfusion. Thus, the $\mathrm{H} / \mathrm{R}$ model was successfully constructed.

\section{Establishment of myocardial I/R model}

A total of 32 C57BL/6 mice (8 weeks old, 23-25 g) were housed in a constant temperature room $\left(22^{\circ} \mathrm{C}, 12 \mathrm{~h}\right.$ light/dark cycle, 50-55\% humidity) with water and food ad libitum. The mice were randomly divided into two groups (sham group and I/R injury group, 16 per group). After adapting to the environment for 5 days, the mice were anesthetized with $1 \%$ pentobarbital sodium $(50 \mathrm{mg} / \mathrm{kg}$ ) intraperitoneal injections before endotracheal intubation. Next, the mice were placed on the operating table in a supine position, had the hair of the neck and chest removed, and were disinfected with iodophor. The limbs were fixed on electrodes to connect to the electrocardiograph (BeneHeart R3, Mindray, China) and a rodent ventilator (R407, RWD Life Science, China) was inserted into the mice mouths, which could monitor the limb lead II electrocardiogram (ECG) with the following conditions: 90-105 times/min respiratory rate, room air supply, and 0.4-0.8 $\mathrm{mL}$ tidal volume. Between the third and fourth ribs, left lateral thoracotomy was carried out. Subsequently, the left anterior descending coronary artery (LADCA) was ligated using $8-0$ atraumatic suture to produce occlusions. When the notable upgrade of ST segment in ECG was recorded by the ECG monitor, the coronary artery was successfully blocked. The suture was released after $30 \mathrm{~min}$ of the block 
and the heart was reperfused, causing an obvious decline in the ST segment. Thus, the myocardial I/R model was successfully constructed. Next, pneumothorax was evacuated manually, and the chest and skin were closed using a 6-0 Prolene suture. For analgesia, buprenorphine $(0.1$ $\mathrm{mg} / \mathrm{kg}$ body weight) was administered ip. Henceforth, the mice were kept in clean cages at room temperature. Additionally, the same procedure except the ligation of LADCA was performed on the sham group of mice.

\section{Determination of myocardial infarct size}

Twenty-four hours after reperfusion, we occluded the LADCA again, and injected $0.2 \mathrm{~mL}$ of $1 \%$ (wt/vol) Evans blue into the aorta. The heart was immediately excised, stored at $-80^{\circ} \mathrm{C}$, cut into $1-\mathrm{mm}$-thick slices, and counterstained with $1 \%$ (wt/vol) TTC (2,3,5-triphenyltetrazolium chloride; Sigma) solution at $37^{\circ} \mathrm{C}$ for $15 \mathrm{~min}$. The sample pictures were captured. Then, the infarct area was assessed using Image-Pro Plus 6.0 software. Myocardial infarct size is reported as (infarct area/whole heart area) $\times 100 \%$.

\section{Quantitative real-time PCR (qRT-PCR)}

Total RNAs from myocardial tissues or cardiomyocytes were extracted using Trizol (Invitrogen). cDNAs were derived from RNA reverse transcription with a test kit (Quanti Tect Reverse Transcription Kit, Qiagen, USA). PCR was conducted with Applied Biosystems ${ }^{\circledR}$ ViiA $^{\text {TM }} 7$ Real-Time PCR System (Life Technologies, USA) using SYBRTM Green PCR Kit (Life Technologies). GAPDH was defined as the internal reference. The $2^{-\Delta \Delta C t}$ method was applied to analyze the relative mRNA expression. The following primers were used: miR-26a-5p forward: 5'-UCC AUA AAG UAG GAA ACA CUA CA-3', reverse: 5'-CAG UAC UUU UGU GUA GUA CAA-3'; PTEN forward: 5'-AAG ACC ATA ACC CAC CAC AGC-3', reverse: 5'-ACC AGT TCG TCC CTT TCC AG-3'; GAPDH forward 5'-GAC TCA TGA CCA CAG TCC ATG C-3', reverse 5'-AGA GGC AGG GAT GAT GTT CTG-3'.

\section{Western blot}

Total protein from myocardial tissues or cardiomyocytes was lysed using lysis buffer on ice. After quantitation of protein using the BCA method, an equal amount of protein $(20 \mu \mathrm{g})$ was separated onto $10 \%$ SDS-PAGE gels, followed by transferring to PVDF membranes (Millipore, USA) by electroelution. Subsequently, $5 \%$ skim milk was utilized to block the membranes for $2 \mathrm{~h}$ at $37^{\circ} \mathrm{C}$. Next, the membranes were cultured with primary antibodies (dilution 1:1000, Cell Signaling Technology, USA) against PTEN, $\mathrm{PI} \mathrm{KK}, \mathrm{AKT}$, and cleaved caspase 3 at $4^{\circ} \mathrm{C}$ overnight, followed by incubating with secondary antibodies conjugated with $\mathrm{HRP}$ for $2 \mathrm{~h}$ at $37^{\circ} \mathrm{C}$. After washing with TBST solution, the protein bands were measured by an enhanced chemiluminescence detection system (Thermo Fisher Scientific). The internal control was actin.

\section{MTT assay}

Forty-eight hours post-transfection, the transfected cardiomyocytes submitted to $\mathrm{H} / \mathrm{R}$ treatment were seeded onto 96-well plates (Corning Life Science, USA), followed by incubation at $37^{\circ} \mathrm{C}$ for $24,48,72$, and $96 \mathrm{~h}$. MTT $(5 \mathrm{mg} / \mathrm{mL}$ ) solution was added to the culture medium and incubated for another $4 \mathrm{~h}$. Subsequently, dimethyl sulfoxide was applied to dissolve the formazan crystals, and absorbance at $490 \mathrm{~nm}$ was determined by a microplate reader (BioTek, USA).

\section{Flow cytometry}

Flow cytometry with an Annexin V/PI kit (BD Bioscience, USA) was utilized to detect cell apoptosis. Briefly, cardiomyocytes were harvested, washed with PBS, followed by re-suspending in $1 \times$ binding buffer. Next, the cardiomyocytes were stained for $30 \mathrm{~min}$ in a darkroom using Annexin $\mathrm{V}$ and $\mathrm{PI}$, and FACS analysis was performed with FACSCalibur (BD Biosciences).

\section{Luciferase reporter assay}

The $3^{\prime}$ UTR fragments of PTEN containing both mutant (MUT) and wild-type (WT) binding sites of miR-26a-5p were amplified by PCR and cloned into the vector pMIRREPOR $^{\mathrm{TM}}$ Luciferase (Promega Corp., USA) to form luciferase reporter vectors. The vectors and miR-26a-5p mimic were co-transfected into primary cardiomyocytes via Lipofectamine 3000 (Invitrogen). Luciferase activity after $24 \mathrm{~h}$ of transfection was determined by a dual luciferase reporter assay kit (Promega) on a Fluoroskan Ascent Type 379 fluorescence plate reader (Thermo), and normalized to renilla luciferase intensity.

\section{Statistical analysis}

Data are reported as means $\pm S D$ and were assessed by GraphPad Prism Software (version 7, USA). Each experiment was performed repeatedly at least three times. The difference between two groups was evaluated by Student's $t$-test, and the comparison between more than two groups was analyzed by one-way ANOVA with Bonferroni's multiple comparisons test. $A$ value of $P<0.05$ was regarded as a significant difference.

\section{Results}

\section{Establishment of I/R injury model}

As cell apoptosis is reported as a major pathophysiological mechanism in myocardial I/R injury (21), flow cytometry was utilized to determine the cardiomyocyte apoptosis in the I/R injury model. Figure $1 \mathrm{~A}$ revealed that the apoptosis rate of primary cardiomyocytes under $H / R$ treatment was $12.65 \%$, and the apoptosis rate in the control group was $0.71 \%$. In addition, the apoptosis rate of cardiomyocytes after I/R treatment was $40.24 \%$ and in the sham group it was $8.83 \%$ (Figure 1B). This result was validated by pro-apoptotic protein cleaved caspase 
A

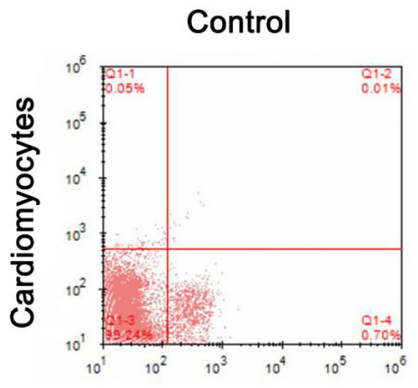

B

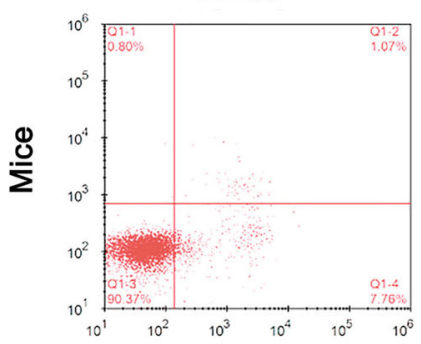

$H / R$ treatment

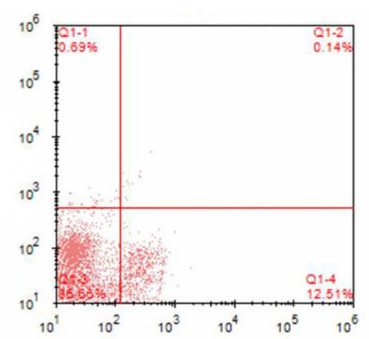

I/R treatment

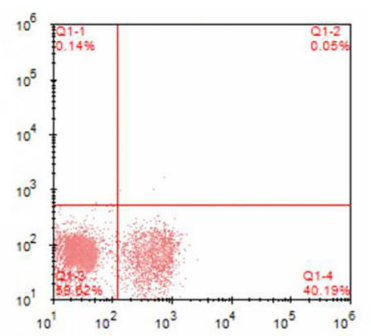

E

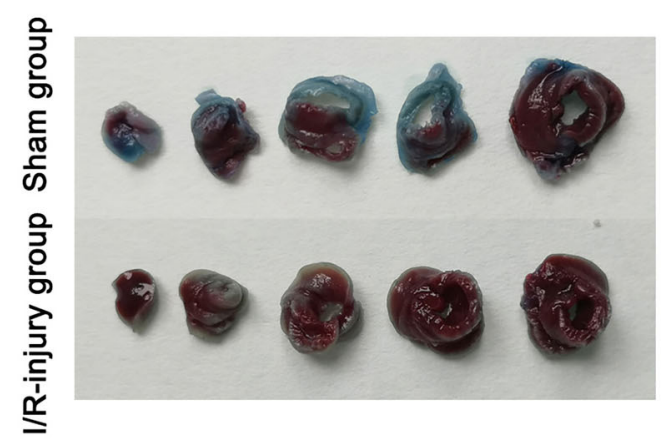

C

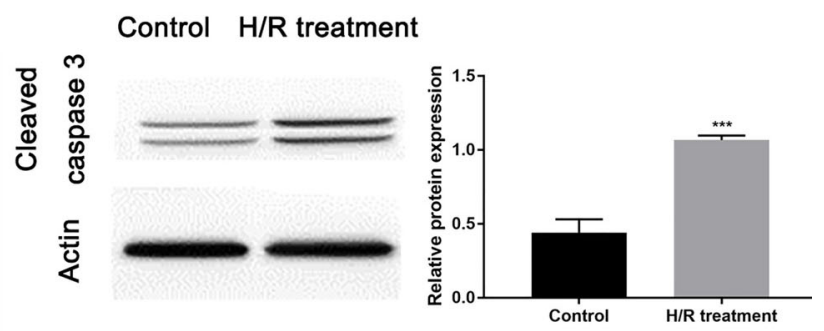

D
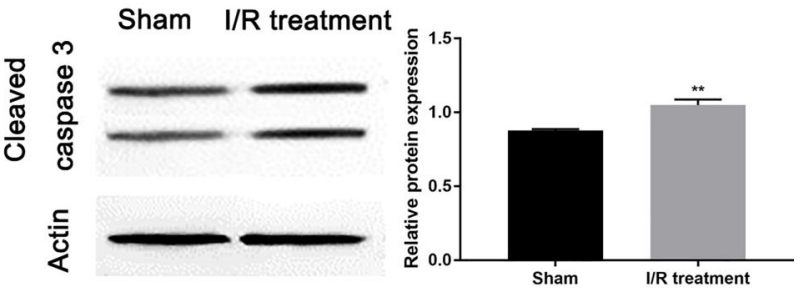

F

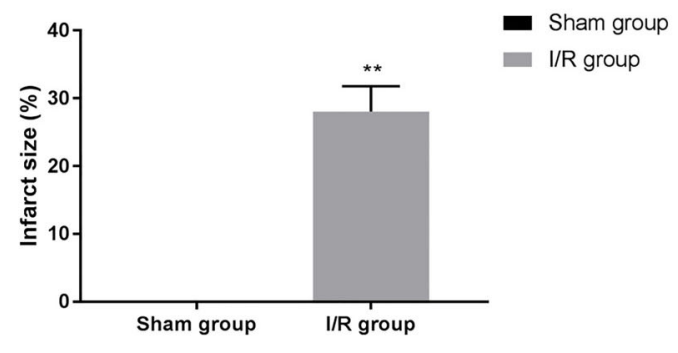

Figure 1. Establishment of ischemia/reperfusion (I/R) injury model. The images of flow cytometry show apoptosis in (A) cardiomyocytes and (B) myocardium of mice upon I/R injury. Western blot examined the expression of cleaved caspase 3 in (C) cardiomyocytes submitted to hypoxia/reoxygenation (H/R) treatment and (D) myocardial tissue upon I/R treatment. E, Representative images of Evans blue/TTC staining in five continuous slices of left ventricle from mice hearts treated with or without I/R treatment. F, The infarct size was quantified by Image-Pro Plus software. Data are reported as means $\pm S D$. ${ }^{* *} P<0.01,{ }^{* \star *} P<0.001$ vs control groups ( $t$-test).

3 expression. Western blot indicated that cleaved caspase 3 level in cardiomyocytes (Figure $1 \mathrm{C}, \mathrm{P}=0.0003$ ) and myocardial tissue (Figure 1D, $P=0.0015$ ) upon $\mathrm{I} / \mathrm{R}$ injury was up-regulated compared to the control groups. Furthermore, Figure $1 \mathrm{E}$ and $\mathrm{F}$ illustrated that there was no infarct damage in the sham group, and yet the infarct size of the myocardium in the I/R injury model was larger than that in the sham group $(P=0.0058)$. Collectively, the $\mathrm{I} / \mathrm{R}$ injury model in vitro and in vivo had been successfully established, and $H / R$ and $I / R$ treatment significantly induced cardiomyocyte apoptosis.

\section{Expression of miR-26a-5p in I/R-injury mice and $H / R$ - induced cardiomyocytes}

To detect miR-26a-5p expression, we applied bioinformatics analysis and qRT-PCR. Firstly, the bioinformatics analysis of miR-26a-5p data from GEO database revealed that its expression was significantly decreased in myocardial I/R injury mice (Figure 2A, $P=0.0004$ ). qRT-PCR indicated that miR-26a-5p expression was considerably down-regulated in primary cardiomyocytes with $H / R$ treatment compared to the control group (Figure 2B, $P=0.0003$ ). Similarly, miR-26a-5p expression showed a 
A

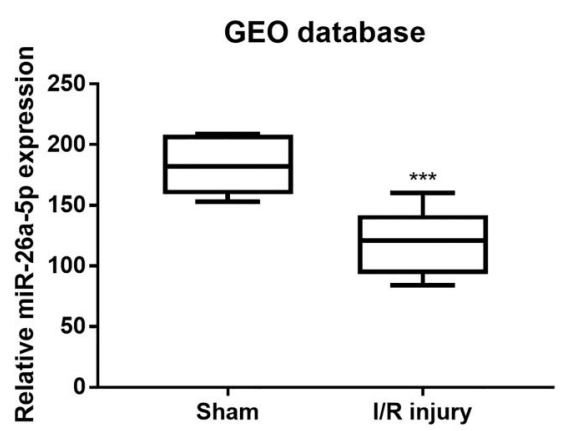

C

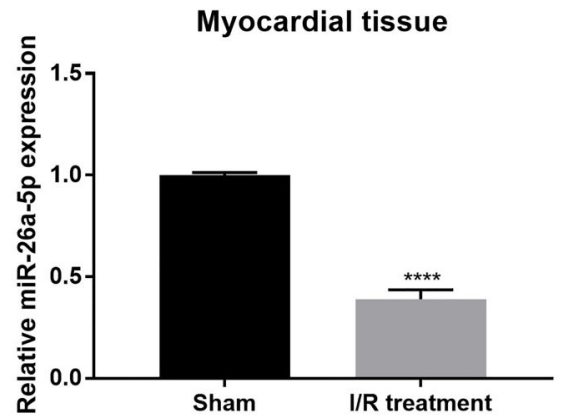

B

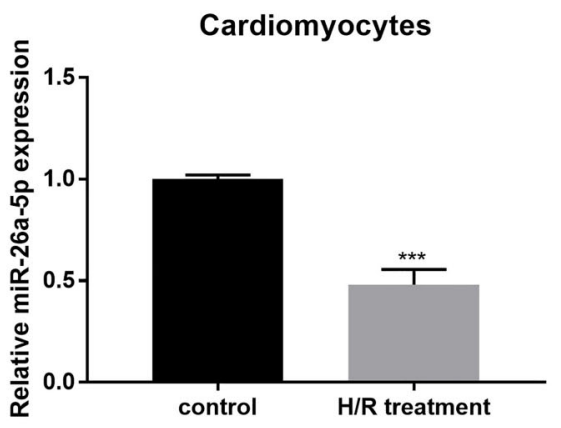

Figure 2. Detection of miR-26a-5p expression. A, The expression data of miR-26a-5p from GEO database were analyzed (medians and interquartile ranges). qRT-PCR was applied to analyze the expression of miR-26a-5p in (B) cardiomyocytes submitted to hypoxia/ reoxygenation $(H / R)$ treatment and $(C)$ myocardial tissue upon ischemia/reperfusion $(I / R)$ injury. Data are reported as means $\pm S D$. ${ }^{* * *} \mathrm{P}<0.001,{ }^{* * *} \mathrm{P}<0.0001$ vs control groups (t-test).

reduction in myocardial tissues of C57BL/6 mice after $\mathrm{I} / \mathrm{R}$ treatment compared to the sham group (Figure 2C, $\mathrm{P}<0.0001)$. Collectively, the expression level of miR-26a$5 p$ was related to $\mathrm{l} / \mathrm{R}$ injury.

Effect of miR-26a-5p on cell viability and apoptosis in $H / R$-induced cardiomyocytes

miR-26a-5p level was greatly increased by miR-26a$5 p$ mimic but decreased by miR-26a- $5 p$ inhibitor, which suggested that transfection was effective (Figure $3 A$, $\mathrm{P}<0.0001)$. Furthermore, MTT assay revealed that miR26a-5p over-expression improved and enhanced the viability of cardiomyocytes, while miR-26a-5p knockdown markedly inhibited cell viability (Figure $3 B$ ). Hence, miR$26 a-5 p$ improved the viability of cardiomyocytes with $I / R$ injury.

After transfection and $\mathrm{H} / \mathrm{R}$ treatment in cardiomyocytes, a lower apoptotic rate of miR-26a-5p mimic group $(7.54 \%)$ was observed compared to miRNA control group (20.86\%), and yet miR-26a-5p inhibitor group had a higher apoptotic rate $(35.89 \%)$ compared to the inhibitor control group (23.25\%) (Figure 3C).

Furthermore, miR-26a-5p over-expression decreased the level of pro-apoptotic protein cleaved caspase 3 , while knockdown of miR-26a-5p increased cleaved caspase 3 level compared to the control group (Figure 3D and E,
$P<0.0001)$. Collectively, miR-26a-5p could inhibit cardiomyocytes apoptosis induced by I/R injury.

\section{Interaction between miR-26a-5p and PTEN}

PTEN was selected as a candidate, and four conserved binding sites of miR-26a-5p were observed in the $3^{\prime}$ UTR of PTEN (Figure 4A). The relationship between PTEN and miR-26a-5p was further validated by luciferase reporter assay. Figure 4B shows that the luciferase activity of the PTEN-WT vector was obviously suppressed by miR-26a-5p compared to the control group ( $P=0.0003)$, while the activity of PTEN-MUT luciferase vector had no significant change between miR-26a-5p mimic transfection group and miRNA control transfection group $(P>0.9999)$. Hence, PTEN was a direct target of miR26a-5p.

After I/R injury treatment, the expression levels of $\mathrm{PTEN}$ in cardiomyocytes (Figure $4 \mathrm{C}, \mathrm{P}=0.0038$; Figure 4E, $P=0.0011$ ) and myocardial tissue (Figure 4D, $P=$ 0.0080; Figure 4F, $\mathrm{P}<0.0001$ ) were up-regulated compared to the control groups. When cardiomyocytes were transfected with miR-26a-5p mimic, miRNA control, miR26a-5p inhibitor, or inhibitor control, miR-26a-5p overexpression greatly decreased PTEN expression, whereas miR-26a-5p knockdown significantly increased PTEN expression compared to the control group (Figure $4 \mathrm{G}$ 
A
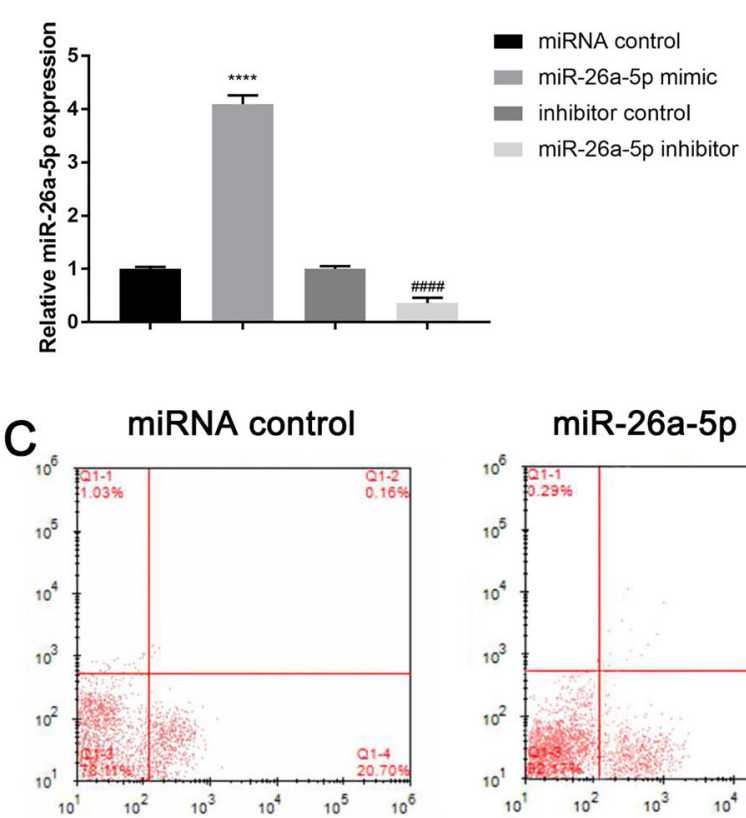

Inhibitor control

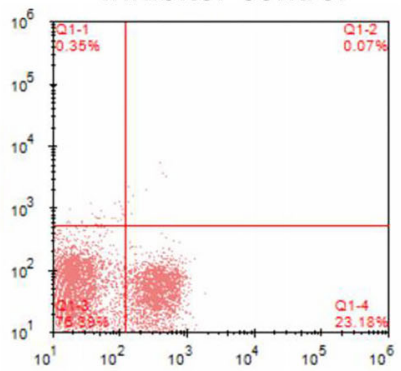

miR-26a-5p mimic

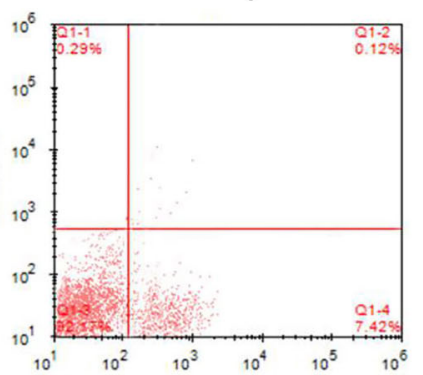

miR-26a-5p inhibitor

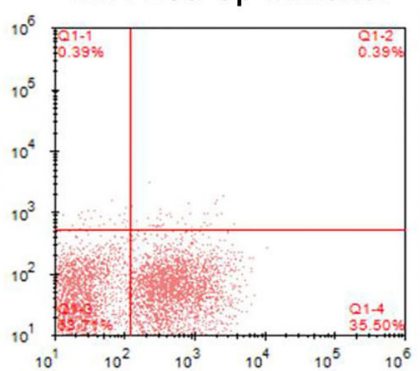

B
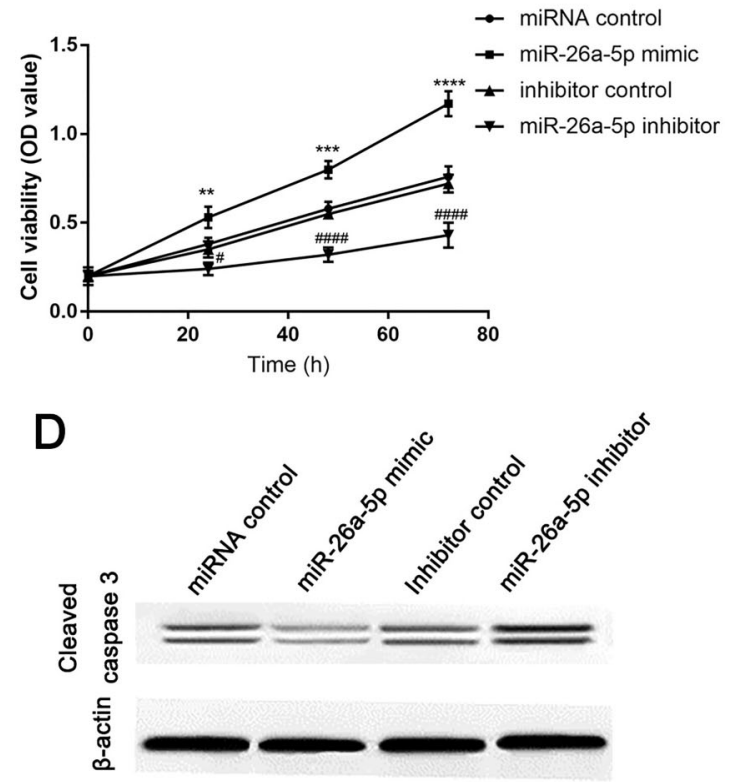

E

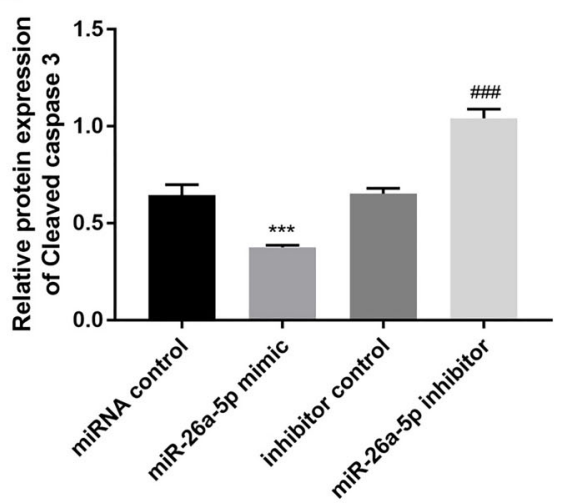

Figure 3. Effect of miR-26a-5p on cell viability and apoptosis. A, After cardiomyocytes were transfected with miR-26a-5p mimic, miRNA control, miR-26a-5p inhibitor, or inhibitor control and treated with hypoxia/reoxygenation, the expression of miR-26a-5p was evaluated by qRT-PCR. The (B) viability and (C) apoptosis of the cells were detected by MTT and flow cytometry, respectively. D, Cleaved caspase 3 expression was assessed by western blot and quantified by ImageJ software $(E)$. Data are reported as means $\pm S D$. ${ }^{*} P<0.01$, ${ }^{* * *} \mathrm{P}<0.001,{ }^{* * \star *} \mathrm{P}<0.0001$ vs miRNA control; ${ }^{\#} \mathrm{P}<0.05,{ }^{\# \# \#} \mathrm{P}<0.001,{ }^{\# \# \#} \mathrm{P}<0.0001$ vs inhibitor control (ANOVA).

and $\mathrm{H}, \mathrm{P}<0.0001)$. Thus, miR-26a-5p could negatively mediate PTEN expression.

Moreover, miR-26a-5p over-expression increased the expression of PI3K and AKT, and yet miR-26a-5p knockdown decreased the level of PI3K and AKT (Figure 4G and $\mathrm{H}, \mathrm{P}<0.0001$ ). As a plethora of studies suggest that PTEN is known as a negative regulator of the PI3K/AKT signaling pathway (22), we speculated that miR-26a-5p promoted the viability of $\mathrm{H} / \mathrm{R}$-induced cardiomyocytes and inhibited apoptosis by inhibiting PTEN expression to activate the PI3K/AKT signaling pathway.

\section{Discussion}

Although reperfusion is currently the most effective treatment for AMI, it still causes a series of additional clinical damage (23). Thus, exploring the molecular mechanism is vital to conquer myocardial I/R injury (24). Like most miRNAs, miR-26a-5p functions in a diversity of biological processes such as cell apoptosis and metastasis $(15,16)$. Additionally, up-regulation of miR-26a-5p in AMI ameliorates cardiac function and suppresses myocardial inflammation (17). miR-26a-5p can also inhibit the 
A

PTEN (41-47) 5'...ACACCAUGaAaAUAAACUUGAaU...3' miR-26a-5p 3' UCGgAUAGGACCUAAUGAACUU $5^{\prime}$

PTEN (1261-1268) 5'...ACUGUUAGGGAAUUUUACUUGAA....3' miR-26a-5p 3' UCGGAUAGGACCUAAUGAACUU 5'

PTEN (2619-2626) 5'...UUACAUGUCUGAAGUUACUUGAA...3' miR-26a-5p 3' UCGgAUAGGACCUAAUGAACUU 5'

PTEN (3800-3807) 5'...CUAAAGGACUUUUUGUACUUGAA...3' miR-26a-5p 3' UCGGAUAGGACCUAAUGAACUU $5{ }^{\prime}$

\section{C}

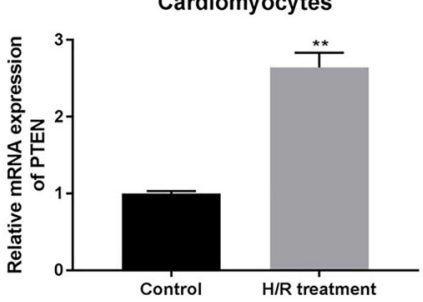

D
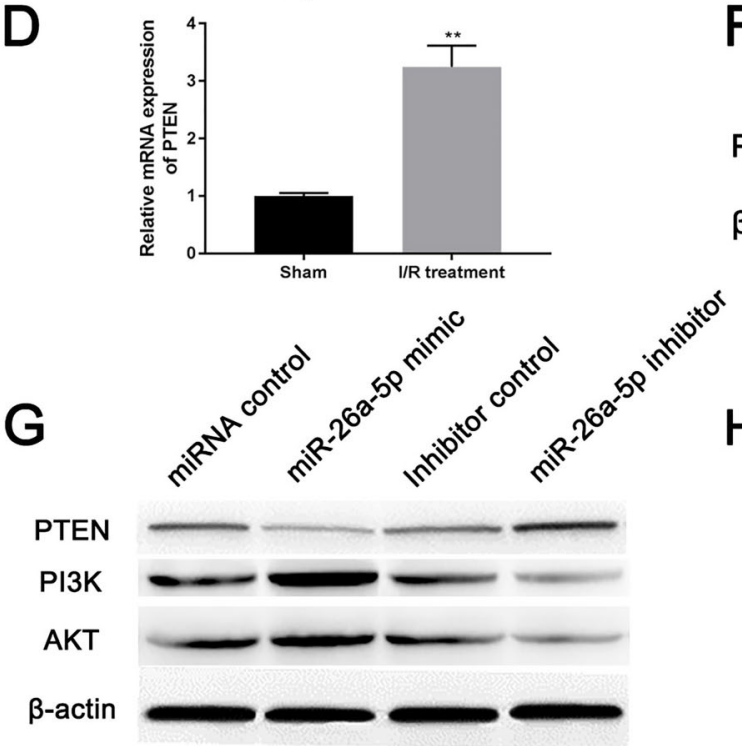

B

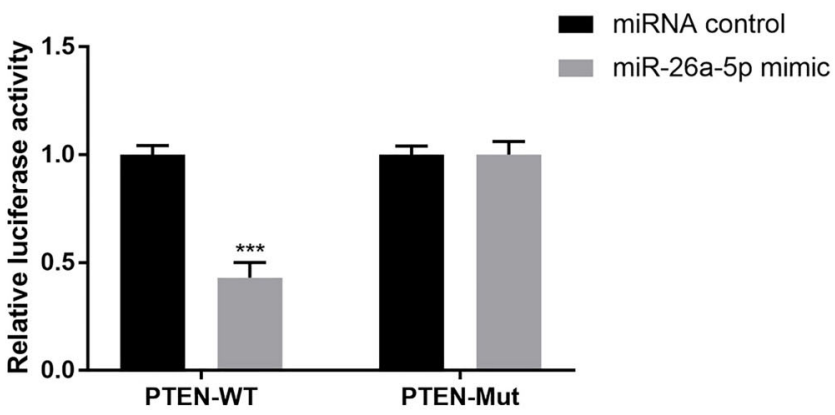

$E$

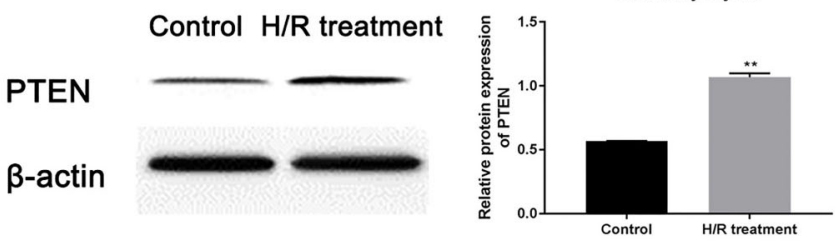

$\mathbf{F}$

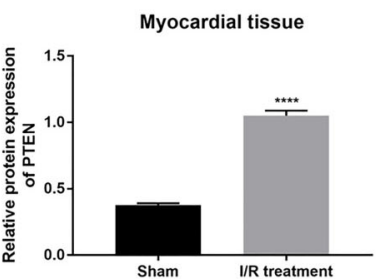

$\mathrm{H}$

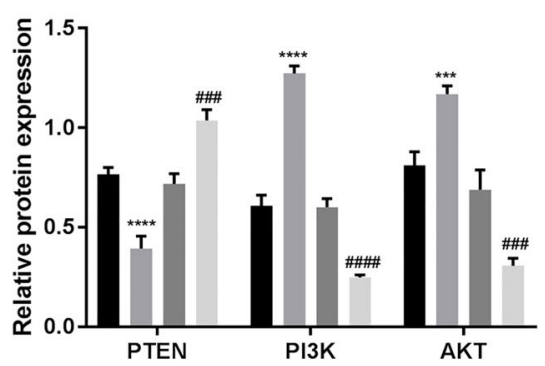

Figure 4. Interaction between miR-26a-5p and PTEN. A, Binding sites between miR-26a-5p and PTEN. B, Luciferase reporter assay measured the luciferase activity of PTEN-WT (wild type) or PTEN-Mut (mutant) vector. The mRNA and protein expression of PTEN in (C and $\mathbf{E}$ ) cardiomyocytes after hypoxia/reoxygenation $(H / R)$ and (D and $\mathbf{F})$ myocardial tissue upon ischemia/reperfusion (l/R) injury was measured by qRT-PCR and western blot, respectively. After transfection of four different miR-26a-5p vectors, the expression of PTEN, $\mathrm{PI} 3 \mathrm{~K}$, and AKT was evaluated by $(\mathbf{G})$ western blot and quantified by $(\mathbf{H})$ ImageJ software. Data are reported as means $\pm \mathrm{SD}$. ${ }^{*} \mathrm{P}<0.01$,

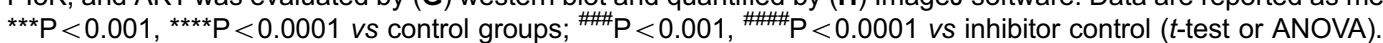

autophagy in cardiac fibroblasts through targeting ULK1, which is critical for cardiac hypertrophy and heart failure (18). Given the effect of miR-26a-5p in cardiovascular diseases, we attempted to study whether miR-26a-5p had an important role in myocardial I/R injury. Our study indicated that the expression of miR-26a-5p was significantly decreased in cardiomyocytes and myocardial tissue upon I/R injury compared to normal cardiomyocytes and myocardial tissue. Besides, the results in this study showed that up-regulation of miR-26a-5p significantly 
improved the viability of cardiomyocytes upon I/R injury, while down-regulation of miR-26a-5p obviously inhibited the cell viability. Moreover, miR-26a-5p over-expression greatly suppressed the cardiomyocyte apoptosis induced by $\mathrm{I} / \mathrm{R}$ injury. Hence, miR-26a-5p could protect cardiomyocytes from I/R injury.

MicroRNAs are key actors in post-transcriptional regulation through regulating the expression of their mRNAs (25). At present, miR-26a-5p has been proven to control its gene targets and to be involved in cancer biology $(15,16)$. For instance, miR-26a-5p facilitates the metastasis of lung cancer cells through targeting ITG $\beta 8$ (26). However, the mechanism of miR-26a-5p target regulation in AMl is not yet reported. Thus, the TargetScan website was used in this study to find the gene targets of miR-26a$5 p$, and PTEN was selected as a potential candidate. Compared with normal cardiomyocytes and myocardial tissue, our study proved that PTEN was over-expressed in cardiomyocytes and myocardial tissue upon I/R injury. Moreover, PTEN was observed to be negatively regulated by miR-26a-5p. PTEN, also named as MMAC1 or TEP1, is known as a tumor suppressor (27). PTEN can oppose the activation of the PI3K/AKT signaling pathway that plays an important role in cell survival, growth, metastasis, and metabolism (28). Therefore, we speculated that miR-

\section{References}

1. Anderson JL, Morrow DA. Acute myocardial infarction. $N$ Engl J Med 2017; 376: 2053-2064, doi: 10.1056/NEJMra 1606915.

2. Ferdinandy P, Hausenloy DJ, Heusch G, Baxter GF, Schulz R. Interaction of risk factors, comorbidities, and comedications with ischemia/reperfusion injury and cardioprotection by preconditioning, postconditioning, and remote conditioning. Pharmacol Rev 2014; 66: 1142-1174, doi: 10.1124/ pr. 113.008300 .

3. Ibanez B, Heusch G, Ovize M, Van de Werf F. Evolving therapies for myocardial ischemia/reperfusion injury. $\mathrm{J} \mathrm{Am}$ Coll Cardiol 2015; 65: 1454-1471, doi: 10.1016/j.jacc.2015. 02.032 .

4. Hausenloy DJ, Yellon DM. Myocardial ischemia-reperfusion injury: a neglected therapeutic target. J Clin Invest 2013; 123: $92-100$, doi: $10.1172 / \mathrm{JCl} 62874$.

5. Frank A, Bonney M, Bonney S, Weitzel L, Koeppen M, Eckle T. Myocardial ischemia reperfusion injury: from basic science to clinical bedside. Semin Cardiothorac Vasc Anesth 2012; 16: 123-132, doi: 10.1177/10892532114 36350.

6. Ferdinandy P, Schulz R, Baxter GF. Interaction of cardiovascular risk factors with myocardial ischemia/reperfusion injury, preconditioning, and postconditioning. Pharmacol Rev 2007; 59: 418-458, doi: 10.1124/pr.107.06002.

7. Davidson SM, Ferdinandy $P$, Andreadou I, Botker HE, Heusch G, Ibanez B, et al. Multitarget strategies to reduce myocardial ischemia/reperfusion injury: JACC review topic of the week. J Am Coll Cardiol 2019; 73: 89-99, doi: 10.1016/j.jacc.2018.09.086. 26a-5p improved viability and suppressed apoptosis in cardiomyocytes upon I/R injury through inhibiting PTEN expression to activate the PI3K/AKT signaling pathway.

In conclusion, miR-26a-5p could effectively protect against myocardial I/R injury through controlling the PTEN/ $\mathrm{PI} 3 \mathrm{~K} / \mathrm{AKT}$ signaling pathway. The relationship among miR26a-5p, PTEN, and PI3K/AKT pathway may offer a new insight for understanding the molecular mechanism of myocardial I/R injury, and provide a potential approach for treatment. However, this was a preliminary study and the pathogenesis of myocardial I/R injury is complex. Thus, large-scale studies will be needed to confirm the detailed mechanisms of miR-26a-5p, PTEN, and PI3K/AKT pathway in myocardial $I / R$ injury through animal experiments and clinical tests in the future.

\section{Acknowledgments}

This work was supported by the Youth Fund of the Second Hospital of Shandong University, China (Y2013 010015); Key Research and Development Project in Shandong, China (2017G006027); Shandong Medical and Health Science and Technology Development Program Project, China (2013WS0243); Shandong Provincial Natural Science Foundation, China (ZR2016HP01).

8. Fabris L, Ceder Y, Chinnaiyan AM, Jenster GW, Sorensen $\mathrm{KD}$, Tomlins $\mathrm{S}$, et al. The potential of MicroRNAs as prostate cancer biomarkers. Eur Urol 2016; 70: 312-322, doi: 10. 1016/j.eururo.2015.12.054

9. Gandellini P, Doldi V, Zaffaroni N. microRNAs as players and signals in the metastatic cascade: Implications for the development of novel anti-metastatic therapies. Semin Cancer Biol 2017; 44: 132-140, doi: 10.1016/j.semcancer. 2017.03.005.

10. Chhabra R, Dubey R, Saini N. Cooperative and individualistic functions of the microRNAs in the miR-23a $27 a \sim 24-$ 2 cluster and its implication in human diseases. Mol Cancer 2010; 9: 232, doi: 10.1186/1476-4598-9-232.

11. Pers YM, Jorgensen C. MicroRNA in 2012: Biotherapeutic potential of microRNAs in rheumatic diseases. Nat Rev Rheumatol 2013; 9: 76-78, doi: 10.1038/nrrheum.2012.236.

12. Hayes J, Peruzzi PP, Lawler S. MicroRNAs in cancer: biomarkers, functions and therapy. Trends Mol Med 2014; 20: 460-469, doi: 10.1016/j.molmed.2014.06.005.

13. Deng Z, He Y, Yang X, Shi H, Shi A, Lu L, et al. MicroRNA29: a crucial player in fibrotic disease. Mol Diagn Ther 2017; 21: 285-294, doi: 10.1007/s40291-016-0253-9.

14. Heggermont WA, Papageorgiou AP, Quaegebeur A, Deckx S, Carai P, Verhesen W, et al. Inhibition of MicroRNA-146a and overexpression of its target dihydrolipoyl succinyltransferase protect against pressure overload-induced cardiac hypertrophy and dysfunction. Circulation 2017; 136: 747-761, doi: 10.1161/CIRCULATIONAHA.116.024171.

15. Wang $\mathrm{H}, \mathrm{Hu} Z$, Chen L. Enhanced plasma miR-26a-5p promotes the progression of bladder cancer via targeting 
PTEN. Oncol Lett 2018; 16: 4223-4228, doi: 10.3892/ol. 2018.9163.

16. Chang L, Li K, Guo T. miR-26a-5p suppresses tumor metastasis by regulating EMT and is associated with prognosis in HCC. Clin Transl Oncol 2017; 19: 695-703, doi: 10.1007/s12094-016-1582-1.

17. Kong B, Qin Z, Ye Z, Yang X, Li L, Su Q. microRNA-26a-5p affects myocardial injury induced by coronary microembolization by modulating HMGA1. J Cell Biochem 2019; 120: 10756-10766, doi: 10.1002/jcb.28367.

18. Zheng L, Lin S, Lv C. MiR-26a-5p regulates cardiac fibroblasts collagen expression by targeting ULK1. Sci Rep 2018; 8: 2104, doi: 10.1038/s41598-018-20561-4.

19. Feng $\mathrm{Y}$, Zou L, Yan D, Chen H, Xu G, Jian W, et al. Extracellular microRNAs induce potent innate immune responses via TLR7/MyD88-dependent mechanisms. J Immunol 2017; 199: 2106-2117, doi: 10.4049/jimmunol. 1700730.

20. Greenberg CC, Connelly PS, Daniels MP, Horowits R. Krp1 (Sarcosin) promotes lateral fusion of myofibril assembly intermediates in cultured mouse cardiomyocytes. Exp Cell Res 2008; 314: 1177-1191, doi: 10.1016/j.yexcr.2007. 12.009 .

21. McCully JD, Wakiyama H, Hsieh YJ, Jones M, Levitsky S. Differential contribution of necrosis and apoptosis in myocardial ischemia-reperfusion injury. Am J Physiol Heart
Circ Physiol 2004; 286: H1923-H1935, doi: 10.1152/ ajpheart.00935.2003.

22. Chen CY, Chen J, He L, Stiles BL. PTEN: tumor suppressor and metabolic regulator. Front Endocrinol (Lausanne) 2018; 9: 338, doi: 10.3389/fendo.2018.00338.

23. Yang CF. Clinical manifestations and basic mechanisms of myocardial ischemia/reperfusion injury. Ci Ji Yi Xue Za Zhi 2018; 30: 209-215, doi: 10.4103/tcmj.tcmj_33_18.

24. Tang J, Zhuang S. Histone acetylation and DNA methylation in ischemia/reperfusion injury. Clin Sci (Lond) 2019; 133: 597-609, doi: 10.1042/CS20180465.

25. Reyes-Herrera PH, Ficarra E. One decade of development and evolution of microRNA target prediction algorithms. Genomics Proteomics Bioinformatics 2012; 10: 254-263, doi: 10.1016/j.gpb.2012.10.001.

26. Song Q, Liu B, Li X, Zhang Q, Cao L, Xu M, et al. MiR-26a$5 p$ potentiates metastasis of human lung cancer cells by regulating ITGbeta8- JAK2/STAT3 axis. Biochem Biophys Res Commun 2018; 501: 494-500, doi: 10.1016/j.bbrc. 2018.05.020.

27. Lee YR, Chen M, Pandolfi PP. The functions and regulation of the PTEN tumour suppressor: new modes and prospects. Nat Rev Mol Cell Biol 2018; 19: 547-562, doi: 10.1038/ s41580-018-0015-0.

28. Worby CA, Dixon JE. PTEN. Annu Rev Biochem 2014; 83 : 641-669, doi: 10.1146/annurev-biochem-082411-113907. 